Marvin Schewe*, Mohamed A. A. Ismail und Christian Rembe

\title{
Differentielle Laser-Doppler-Vibrometrie für luftgestützte Vibrationsmessungen mit Drohnen
}

\author{
Differential laser-Doppler vibrometry for airborne vibration measurements with drones
}

DOI 10.1515/teme-2021-0071

Zusammenfassung: Die Vibrationsmessung großer und schwer zugänglicher Konstruktionen ist oftmals mit enormen Aufwand verbunden. Durch die Positionierung des Messstrahls eines Laser-Doppler-Vibrometers mit Hilfe an Drohnen befindlicher Spiegel, sind Schwingungsmessungen an schwer zugänglichen Messpunkten möglich. In diesem Beitrag stellen wir mögliche Messmethoden vor, betrachten auftretende Störeinflüsse und zeigen anhand von Messergebnissen inwieweit diese unterdrückt werden können.

Schlüsselwörter: heterodyne Laser-Doppler-Vibrometrie, differentielles Messen, Drohnen, optische Turbulenz, structural health monitoring

Abstract: Vibration measurements on large and hardto-reach structures are usually challenging and timeconsuming. However, by placing the measurement beam of a laser-Doppler vibrometer with the support of mirrors mounted on drones, vibration measurements at hard-toaccess measuring spots are feasible. In this article, we present possible measurement approaches, analyze disturbing effects and demonstrate on the basis of measurement results to which extent they can be reduced.

Keywords: heterodyne laser-Doppler vibrometry, differential measurement, drones, optical turbulence, structural health monitoring,

\section{Einleitung}

Große Konstruktionen wie Windkrafträder, Brücken und Gebäude werden durch Umwelteinflüsse wie Wetter, Wind

\footnotetext{
*Korrespondenzautor: Marvin Schewe, Institut für Elektrische Informationstechnik, TU Clausthal, Leibnizstraße 28, 38678 Clausthal-Zellerfeld, schewe@iei.tu-clausthal.de

Mohamed A. A. Ismail, Institut für Flugsystemtechnik, Deutsches Zentrum für Luft- und Raumfahrt, Lilienthalplatz 7 . 38108 Braunschweig

Christian Rembe, Institut für Elektrische Informationstechnik, TU Clausthal, Leibnizstraße 28, 38678 Clausthal-Zellerfeld
}

und extremere Faktoren wie Erdbeben oder Überschwemmungen mechanisch belastet. Da ein Materialversagen bei diesen Konstruktionen katastrophale Folgen haben kann, ist es wichtig exakte Aussagen über das Materialverhalten treffen zu können. Hauptsächlich werden dafür Experimente zur Untersuchung der Festigkeit- bzw. Schwingfestigkeit durchgeführt. Darüber hinaus ist die Kontrolle des aktuellen Zustands eines Materials ebenfalls ein wichtiges Mittel, um beginnende Materialschäden zu erkennen und ein katastrophales Materialversagen zu verhindern. Eine Möglichkeit Schäden frühzeitig und zerstörungsfrei zu erkennen, ist die Untersuchung des Schwingverhaltens dieser Objekte, da sich dadurch bereits kleinste Schäden detektieren lassen [1]. Dafür eignen sich grundsätzlich Dehnungsmessstreifen (DMS) oder Laser-Doppler-Vibrometer (LDV), wie z.B. in [2] beschrieben. Da bei großen zu untersuchenden Objekten eine Montage von DMS mit hohem Aufwand verbunden ist, bietet sich die kontaktlose Untersuchung durch LDV eher an. Dabei ist die Ausrichtung des Laserstrahls des LDV bei großen Objekten allerdings nur eingeschränkt möglich. Beispielsweise können die Einfallswinkel ungünstig sein, was zu einer schlechten Rückstreuung führt, oder Bereiche von Interesse sind verdeckt, wodurch diese auf optischen Wege messtechnisch nicht direkt zugänglich sind. In der Literatur finden sich diesbezüglich bereits Ansätze LDV und Drohnen zu kombinieren, um fliegende LDV zu konstruieren, die somit an beliebige Positionen gebracht werden können [3]. Dabei muss die Relativbewegung der Drohne kompensiert werden und es müssen weitere Probleme wie die Spannungsversorgung oder die Datenübertragung betrachtet werden. Ansätze zur Kompensation der Relativbewegung wurden bereits beschrieben [3]. Ein weiterer Ansatz ist die Nutzung eines am Boden positionierten LDV und die Verwendung von Drohnen, an denen Spiegel befestigt sind, über die der Laserstrahl des LDV auf dem Messobjekt positioniert werden kann. Für diesen Ansatz haben wir die grundsätzliche Möglichkeit beschrieben und bereits erste Ergebnisse gezeigt [5]. Vorteilhaft an dieser Methode ist, dass an einem bestehenden LDV, sowie der Spannungsversorgung und Datenübertragung, keine großen Änderungen vorgenommen werden müssen. 
Allerdings müssen geeignete Methoden verwendet werden, die eine zuverlässige Positionierung des Lasers auf der Drohne, und darüber auf dem Messobjekt, ermöglichen können. Bei diesen Messungen kann das Messsignal durch die Vibrationen der Drohnenrotoren, sowie durch Umwelteinflüsse wie Wind und Hitzeflimmern gestört werden und den Rauschpegel des Signals signifikant erhöhen.

Zur Verringerung des Einflusses dieser Störungen, kann ein differentiell messendes LDV (DLDV) verwendet werden. Das Potential dafür haben wir mit einem von uns entwickelten DLDV durch die Unterdrückung des Einflusses von Hitzeflimmern auf den Rauschpegel bereits aufgezeigt [4]. Des weiteren konnten wir bereits die erfolgreiche Unterdrückung der Schwingungsfrequenzen der Rotoren einer Drohne zeigen [5]. Das Grundprinzip einer differentiellen Messung ist, dass sowohl der Mess- als auch der Referenzstrahl durch auftretende Störungen geführt werden und diese somit die gleichen Störungen erfahren und diese dadurch kompensiert werden können [4].

\section{Methoden}

Im Folgenden werden Experimente zur Untersuchung inwieweit und unter welchen Bedingungen der Einfluss von Umwelteinflüssen und der Vibration der Drohne auf den Rauschpegel verringert werden kann, vorgestellt. Diese Experimente sollen ohne ein Positionierungssystem möglich sein, weshalb die Drohne für unsere Experimente mechanisch fest mit dem Boden verbunden ist.

In Abb. 1 ist ein schematischer Aufbau einer Messung gezeigt. Der Messstrahl des DLDV wird mithilfe eines Spiegels, der am Kameragimbal der Drohne befestigt ist, auf ein Messobjekt positioniert. Der Referenzstrahl wird auf einen am Kameragimbal befestigen Reflektor positioniert und durchläuft so die Turbulenz $T_{1}$. Um eine Verbesserung des Rauschpegels durch eine Unterdrückung der auftretenden Störungen nachzuweisen, wird jeweils eine LDV-Referenzmessung aufgenommen. Für diese Messung wird der Referenzstrahl nicht auf den Reflektor der Drohne positioniert, sondern auf einem räumlich getrennten Reflektor.

Die Umwelteinflüsse sollen durch zwei Turbulenzen $T_{1}$ und $T_{2}$ simuliert werden. Abb. 1 zeigt ein Beispiel für eine im Labor erstellte Turbulenz. Um reale Effekte nachzubilden, werden die Winkel und Positionen der Elemente (zwei Lüfter und ein Heizelement) variiert. Zusätzlich führt die Rotation der Propeller der Drohne im aktiven Zustand zu einer Turbulenz.

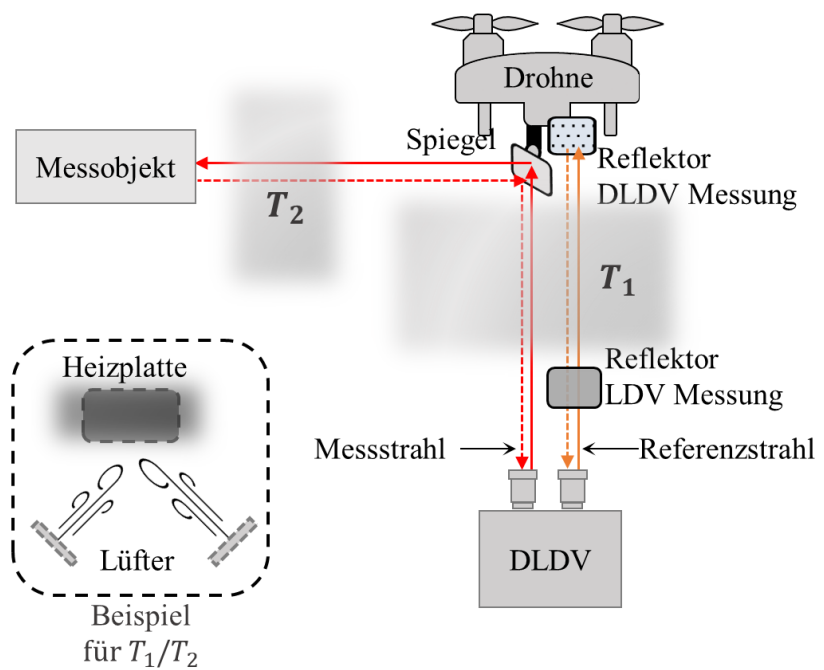

Abb. 1: Grundprinzip einer differentiellen LDV-Messung an einem Messobjekt über eine Drohne.

Um die resultierenden Rauschpegel besser beurteilen und die jeweiligen Einflüsse genau zuordnen zu können, wurden diverse Vergleichsmessungen aufgenommen, bei denen der Aufbau teilweise stark von dem des in Abb. 1 dargestellten abweicht. Relevant ist bei jeder Messung die Position des Mess- sowie Referenzstrahls. Zur Veranschaulichung wird jeweils ein Piktogramm des Aufbaus einer DLDV-Messung neben den Frequenzspektren gezeigt. Für die entsprechende LDV-Referenzmessung wird der Referenzstrahl auf einem räumlich getrennten Reflektor positioniert.

\section{Ergebnisse}

\subsection{Experimente}

\subsubsection{Grundrauschpegel}

Die erste Referenzmessung wurde auf Reflektorfolie auf einem schwingungsgedämpften Labortisch aufgenommen, um den Einfluss anderer Faktoren möglichst gering zu halten und Kopplungen zu verringern. Die zugehörigen Frequenzspektren des Grundrauschpegels einer LDV- und DLDV-Messung sind in Abb. 2 dargestellt. Bereits am Grundrauschpegel ist ein geringerer Rauschpegel der DLDV-Messung zu erkennen. Die in der LDV-Messung dominanten Ausschläge von 0-50 Hz lassen sich primär auf Gebäudeschwingungen zurückführen. Diese Schwingungen können bei einer differentiellen Messung auf dem gleichen Messobjekt nur anteilig unterdrückt werden. 

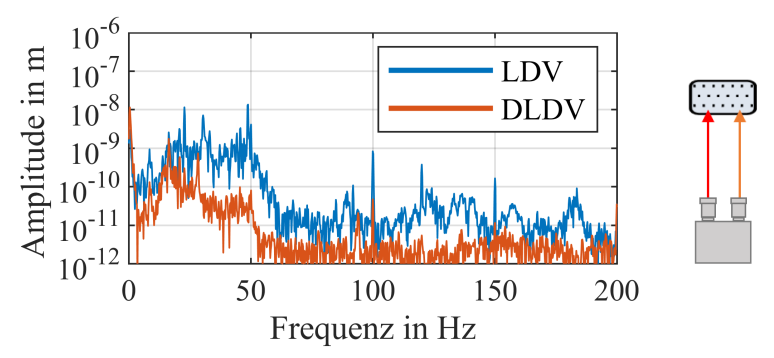

Abb. 2: Grundrauschpegel einer LDV- und einer DLDV-Messung auf Reflektorfolie auf einem schwingungsgedämpften Labortisch; $\mathrm{RBW}=0,2 \mathrm{~Hz}$.

\subsubsection{Einfluss von Turbulenzen}

Die grundsätzliche Funktionalität der Unterdrückung des Einflusses von Störungen haben wir bereits in [5] gezeigt. In den Abb. 3 und 4 sind zwei repräsentative Frequenzspektren von zwei Messungen auf dem Labortisch auf Reflektorfolie gezeigt. Je turbulenter die Störung ist, desto schlechter ist die Unterdrückung des auftretenden Rauschens. Der Einfluss eines oder zwei Lüfter, die in die gleiche Richtung ausgerichtet sind, kann nahezu vollständig kompensiert werden, wie in Abb. 3 zu sehen ist. Abb. 4 zeigt den Fall einer höheren Turbulenz durch zwei
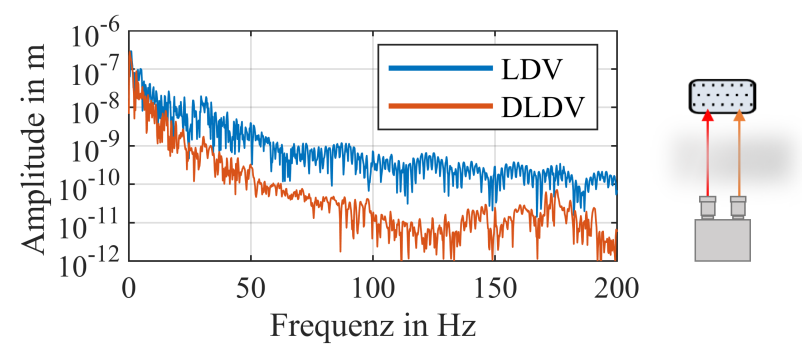

Abb. 3: Vergleich der Frequenzspektren einer LDV und DLDVMessung mit Turbulenz $T_{1}$ (zwei Lüfter mit gleicher Orientierung und einer Heizplatte); RBW $=0,2 \mathrm{~Hz}$.

zueinander ausgerichtete Lüfter. Die unterschiedlich auftretenden Störungen an beiden Strahlen können so nicht vollständig unterdrückt werden.

Um ein reproduzierbares, starkes und dynamisches Ereignis zu erzeugen, wurde zusätzlich eine Messung auf dem gedämpften Labortisch aufgenommen, bei der als Störquelle eine Drohne neben dem Tisch positioniert wurde. Die von uns verwendete Drohne durchläuft beim ersten Start der Rotoren einen kurzen Selbsttest, bei dem die Rotoren zweimal kurz, aber mit hoher Drehzahl betrieben werden. Da dieses Ereignis immer gleich abläuft, eignet es sich, um eine LDV- und DLDV-Messung zu vergleichen.

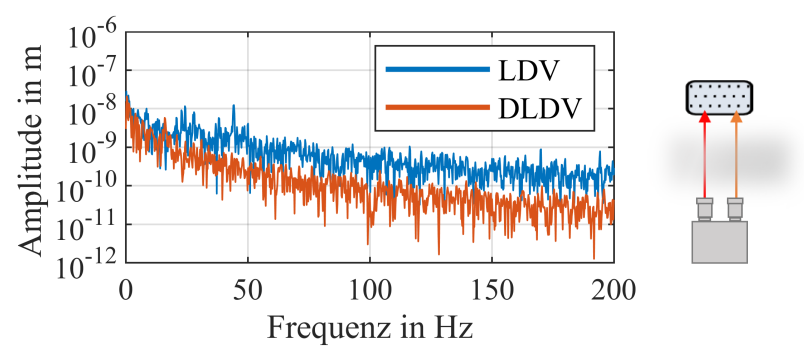

Abb. 4: Vergleich der Frequenzspektren einer LDV und DLDVMessung mit Turbulenz $T_{1}$ (zwei Lüfter zueinander orientiert und einer Heizplatte); RBW $=0,2 \mathrm{~Hz}$.

Die Zeitsignale und die Frequenzspektren dieser Messung sind in Abb. 5 zu sehen. Bei den Messungen starten die
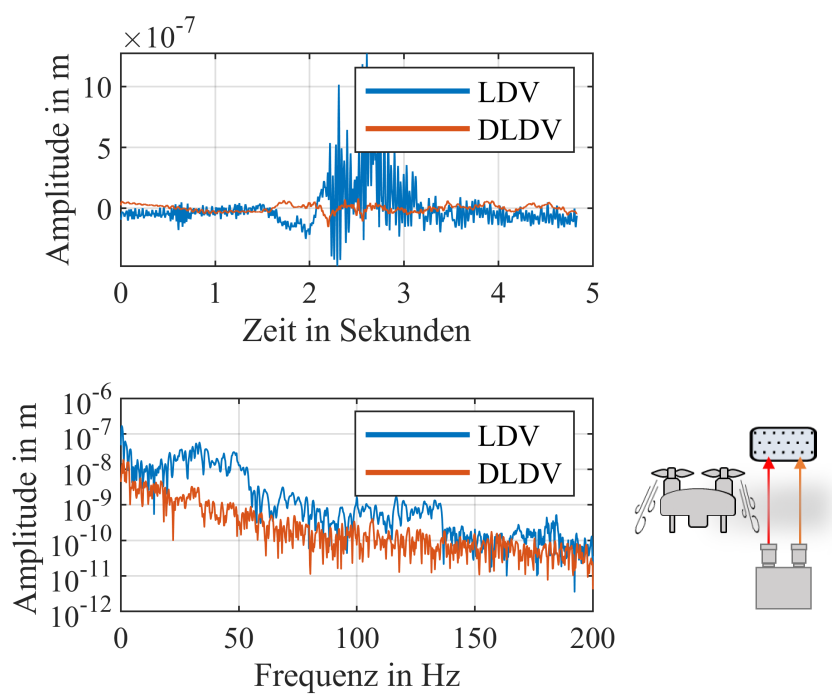

Abb. 5: 42 Vergleich der Zeitsignale und Frequenzspektren einer LDV und DLDV-Messung mit dynamischer Turbulenz durch eine Drohne; $\mathrm{RBW}=0,2 \mathrm{~Hz}$.

Rotoren etwa ab $2 \mathrm{~s}$, die Beschleunigung erfolgt zwischen $2 \mathrm{~s}$ und $3 \mathrm{~s}$. Ab $3 \mathrm{~s}$ drehen die Rotoren im Leerlauf bei ungefähr $900 \mathrm{rpm}$. Sowohl im Zeitsignal als auch im Frequenzspektrum ist zu erkennen, dass bei der differentiellen Messung der Störeinfluss weitgehend unterdrückt werden kann, wohingegen bei einer LDV-Messung starke Ausschläge sichtbar sind.

\subsubsection{Einfluss der Drohnenrotoren}

Für eine Referenzmessung zur Untersuchung des Einflusses der Drohnenrotoren wurde eine Messung auf dem Reflektor auf dem Kameragimbal der Drohne aufgenommen. Bei der DLDV-Messung wurde sowohl Mess- als auch Re- 
ferenzstrahl darauf positioniert. Die Ergebnisse für eine Messung mit ausgeschalteten Rotoren sind in Abb. 6 und die einer Messung mit aktiven Rotoren in Abb. 7 gezeigt.
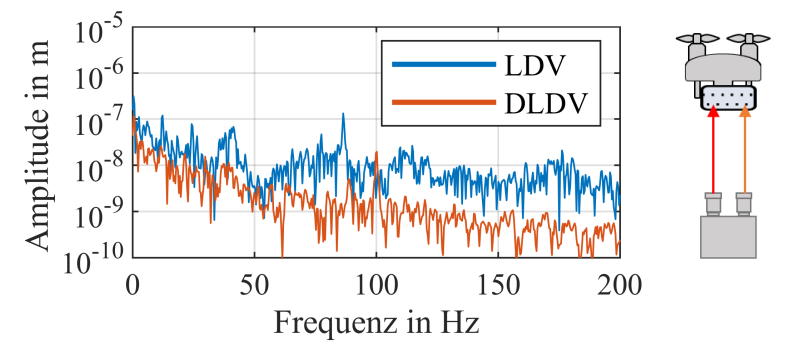

Abb. 6: Vergleich der Frequenzspektren einer LDV- und DLDVMessung auf einem Reflektor auf einem angeschalteten Kameragimbal einer Drohne; RBW $=0,2 \mathrm{~Hz}$.
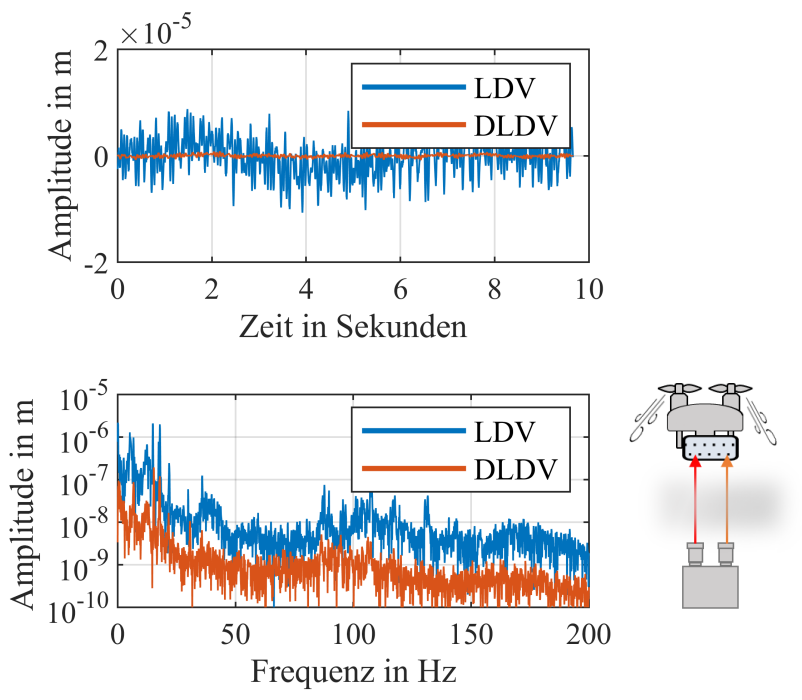

Abb. 7: Vergleich der Zeitsignale und Frequenzspektren einer LDV- und DLDV-Messung auf einem Reflektor auf einem angeschalteten Kameragimbal einer Drohne mit aktiven Rotoren (ca. $900 \mathrm{rpm}) ; \mathrm{RBW}=0,1 \mathrm{~Hz}$.

Der erhöhte Rauschpegel lässt sich auf den größeren Einfluss vorhandener Umgebungsstörungen (Gebäudeschwingungen) zurückführen, die durch die ungedämpfte mechanische Kopplung der Drohne hervorgerufen wird. Ein zusätzlicher Einfluss sind die Bewegungen des Kameragimbals, die sporadisch zur Nachpositionierung erfolgen. Durch diese Bewegungen können die Störungen auch bei der DLDV-Messung schlechter kompensiert werden. Neben der dominanten Rotationsfrequenz der Drohne bei ca. $15 \mathrm{~Hz}-20 \mathrm{~Hz}$, ist auch der gesamte Rauschpegel durch die erzeugten Vibrationen und Turbulenzen deutlich höher. Die Rotationsfrequenz der Drohnenrotoren, sowie der ge- samte Rauschpegel, kann durch eine differentielle Messung, mit Positionierung beider Strahlen auf dem Reflektor, um ungefähr $20 \mathrm{~dB}$ verringert werden.

\subsubsection{Einfluss des Kameragimbals und der Strahlumlenkung}

Sobald der Messstrahl über einen Spiegel auf ein externes Messobjekt abgelenkt wird, erhöht sich der Rauschpegel bereits ohne Turbulenzen deutlich, wie in Abb. 8 dargestellt ist.

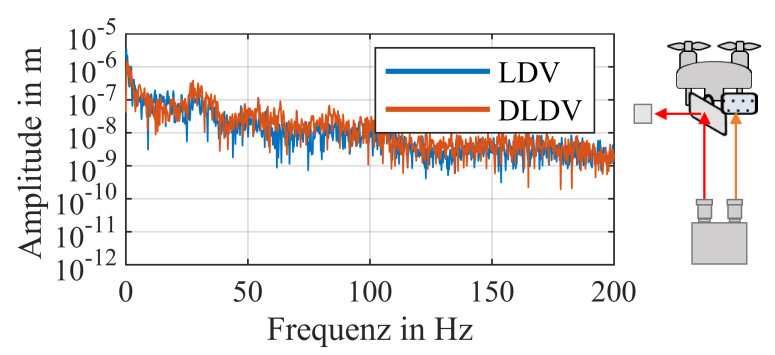

Abb. 8: Vergleich der Frequenzspektren einer LDV- und DLDVMessung auf einem Messobjekt mit Ablenkung des Messstrahls durch einen auf einer Drohne positionierten Spiegel mit Nachregelung des Kameragimbals; RBW $=0,2 \mathrm{~Hz}$.

Durch die hohen Rauschamplituden durch die Bewegungen des Kameragimbals, ist der grundsätzliche Rauschpegel der DLDV- und LDV-Messung nahezu identisch, da beide Strahlen unterschiedliche Störungen erfahren, die nicht kompensiert werden können. Durch den hohen Grundrauschpegel sind positive Kompensationseffekte durch differentielles Messen nicht sichtbar. Bei abgeschalteter Drohne und einem so fixierten Spiegel, der nicht mehr durch den Kameragimbal nachjustiert wird, gibt es einen signifikant geringeren Rauschpegel, wie in Abb. 9 gezeigt ist.

\subsection{Auswertung}

Aus den Referenzmessungen können diverse Informationen gewonnen werden. Bei einer differentiellen Messung mit Positionierung beider Strahlen auf dem gleichen Messobjekt, lässt sich erkennen, dass beim Grundrauschpegel aus Abb. 2 eine Unterdrückung von kleinsten Umgebungseinflüssen, wie Gebäudeschwingungen, erfolgt. Anhand der Abb. 3, 4 und 5 lässt sich eine Unterdrückung stärkerer turbulenter Einflüsse zeigen. Auch eine Unterdrückung der Störeinflüsse der Drohne lässt sich bei einer differentiellen 


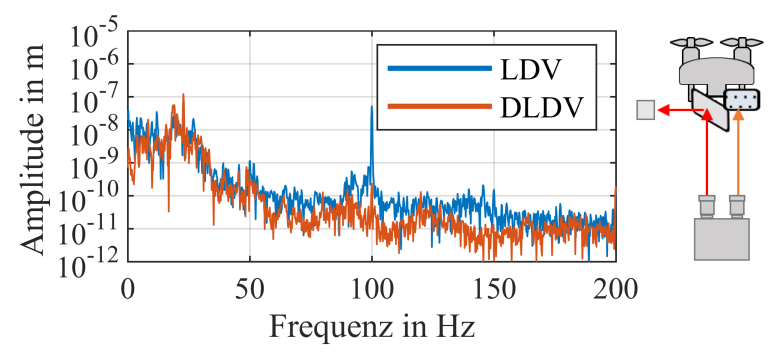

Abb. 9: Vergleich der Frequenzspektren einer LDV- und DLDVMessung auf einem Messobjekt mit Ablenkung des Messstrahls durch einen auf einer Drohne positionierten Spiegel ohne Nachregelung des Kameragimbals; RBW $=0,2 \mathrm{~Hz}$.

Messung nachweisen, wie in den Abb. 6 und 7 gezeigt wird. Durch Abb. 8 und 9 wird stark verdeutlicht, dass der Einfluss der Bewegungen des Kameragimbals einen großen Einfluss hat, da kleinste Bewegungen des Kameragimbals, bei großen Entfernungen des Messobjekts, zu großen Wegen und Störungen durch den Messstrahl führen können. Unabhängig von der Bewegung des Kameragimbals, erfahren der Mess- und Referenzstrahl bei einer Messung über einen Spiegel unterschiedliche Störungen, was zu einem geringeren Unterschied der Rauschpegel von LDV- und DLDV-Messung führt.

Zusammenfassend lässt sich das Potential einer differentiellen Messung auf einem Messobjekt über einen an einer Drohne befestigten Spiegel am leichtesten mit Hilfe der in Abb. 10 gezeigten Messung mit aktiven Rotoren zeigen.

Im Vergleich zu einer Messung mit Positionierung von beiden Laserstrahlen auf der Drohne, wie in Abb. 7, ist der Rauschpegel dieser Messung auch für das DLDV deutlich höher. Bei dieser Messung werden die Turbulenzen $T_{1}$ und $T_{2}$ durch die Drohnenrotoren erzeugt. Durch die Umlenkung des Messstrahls erfährt dieser sowohl auf dem Hinals auch auf dem Rückweg die Vibrationen der Drohne und die Turbulenzen. Bei der LDV-Messung wirkt sich dies bei einer Erhöhung der Rotationsfrequenz zwischen $15-20 \mathrm{~Hz}$ um ca. $3 \mathrm{~dB}$ (Faktor 2) aus. Bei der DLDVMessung kann die Rotationsfrequenz anteilig kompensiert werden, da der Referenzstrahl zumindest auf einem Weg die Störung der Drohnenrotoren und der Turbulenz $T_{1}$ erfährt. Die Amplitude liegt dabei ungefähr auf der einer LDV-Messung auf der Drohne, wie in Abb. 7.

Relevant zum Verständnis sind die Phasenverschiebungen der Strahlen $\varphi_{R, d}(t)$ und $\varphi_{M, d}(t)$ durch den Dopplereffekt sowie durch Störeinflüsse wie Fluktuationen des Brechungsindex der Luft durch Turbulenzen. Bezogen auf eine differentielle Messung mit Ablenkung des Messstrahls auf ein Messobjekt mit aktivierten Rotoren und den Tur-
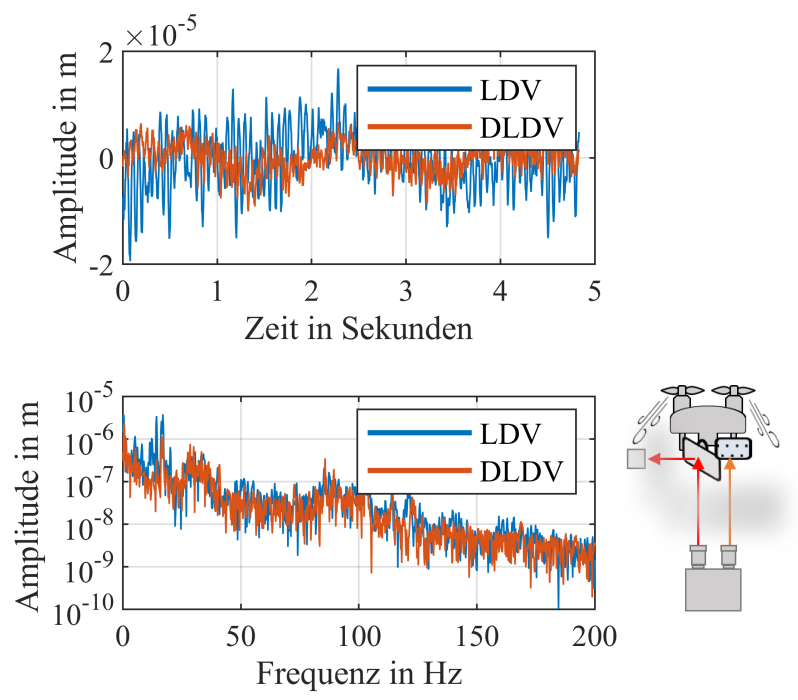

Abb. 10: Vergleich der Zeitsignale und Frequenzspektren einer LDV- und DLDV-Messung auf einem Messobjekt mit Ablenkung des Messstrahls durch einen auf einer Drohne positionierten Spiegel mit aktiven Rotoren der Drohne im Leerlauf (ca. 900 rpm); $\mathrm{RBW}=0,2 \mathrm{~Hz}$.

bulenzen $T_{1}$ und $T_{2}$, wie in Abb. 10, ergibt sich

$$
\begin{aligned}
\varphi_{R, d}= & 2 \varphi_{T, 1}+\varphi_{D, \text { high }}+\varphi_{D, \text { low }}+\varphi_{\mathrm{G}, 1} \\
\varphi_{M, d}(t)= & 2 \varphi_{T, 1}+2 \varphi_{D, \text { high }}+\varphi_{D, \text { low }}+\varphi_{\mathrm{G}, 2} \\
& +\varphi_{d, O}+2 \varphi_{T, 2}
\end{aligned}
$$

mit den Phasenverschiebungen $\varphi_{T, 1}$ und $\varphi_{T, 2}$ durch die Turbulenzen $T_{1}$ und $T_{2}, \varphi_{D}$, high durch die hochfrequente Rotationsfrequenz, $\varphi_{D \text {, low }}$ durch die niederfrequente, vertikale Bewegung der Drohne $\varphi_{d, O}$ durch das Messobjekt und $\varphi_{G, 1}$ sowie $\varphi_{G, 2}$ durch unterschiedliche Störungen der Strahlen durch Bewegungen des Kameragimbals, die zu $\varphi_{G}=\varphi_{G, 1}+\varphi_{G, 2}$ kombiniert werden. Die kombinierte Phasenverschiebung für eine DLDV-Messung $\varphi_{d \text {,DLDV }}$ resultiert aus der Differenz von $\varphi_{M, d}$ und $\varphi_{R, d}$ und ist

$$
\varphi_{d, \mathrm{DLDV}}=\varphi_{d, D, \mathrm{high}}+\varphi_{d, O}+2 \varphi_{T, 2}+\varphi_{\mathrm{G}} .
$$

Die Phasenverschiebung für eine LDV-Messung $\varphi_{d, \mathrm{LDV}}$ entspricht der Phasenverschiebung $\varphi_{M, d}$ aus Gl. $1 \mathrm{~b}$.

\subsubsection{Diskussion}

Mit dem in Abb. 1 gezeigten Aufbau einer differentiellen Messung lassen sich nur die Turbulenzen $T_{1}$ unterdrücken und die Amplitude der Störung der Drohnenrotoren halbieren, wie aus Gl. 1b und Gl. 2 hervorgeht.

Um auch die Turbulenzen $T_{2}$ und die Rotationsfrequenz vollständig zu unterdrücken, ist ein Aufbau wie in Abb. 11 dargestellt, möglich. 


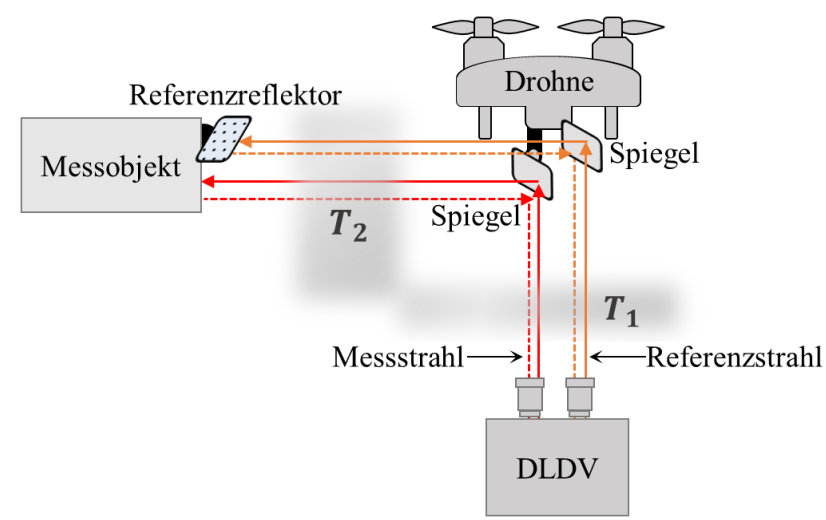

Abb. 11: Grundprinzip einer DLDV-Messung an einem Messobjekt über eine Drohne mit einem schwingungsgedämpften Referenzreflektor für den Referenzstrahl.

Durch die Positionierung des Referenzstrahls über einen Spiegel und Verwendung eines Referenzreflektors, damit Mess- und Referenzstrahl die gleichen Störungen erfahren, wird eine hohe Reflektivität garantiert und sichergestellt, dass Referenzlicht zurückreflektiert wird. Der erhöhte Messaufwand ist dabei weiterhin deutlich geringer als die Montage eines DMS. Denkbar ist beispielsweise eine flexible Befestigung eines Referenzreflektors am Messobjekt mit Hilfe einer Drohne.

Eine alternative Herangehensweise ist die Verwendung von zwei LDVs für die differentielle Messung. Dabei ist eine unabhängige elektronische Verarbeitung des Mess- und Referenzstrahls möglich, wodurch eine vollständige Unterdrückung der Rotationsfrequenz erreichbar sein könnte. Allerdings ist bei diesem Ansatz eine aufwändigere Datenverarbeitung notwendig und es ist mit einem höheren Grundrauschpegel zu rechnen.

\section{Zusammenfassung}

Durch die vorgestellten Experimente in Kapitel 2 kann eine Aussage über die Einflüsse der einzelnen Teilaspekte einer luftgestützten Vibrationsmessung durch Drohnen mit einem (D)LDV getroffen werden.

Wir konnten unsere Ergebnisse aus [4] und [5] zur Unterdrückung von Hitzeflimmern und der Vibration von Drohnenrotoren durch ein DLDV verifizieren und auf zufällige Turbulenzen, die durch Wärme und Luftströme erzeugt werden, erweitern. Dadurch ist eine Anwendung im Freien zur Unterdrückung beliebiger Umwelteinflüsse durch ein DLDV naheliegend. Dabei ist auf einen geringen Abstand des Mess- und Referenzstrahls zueinander zu achten [4].
Im spezifischen Anwendungsfall der Positionierung des Messstrahls eines (D)LDV über einen am Kameragimbal einer Drohne befindlichen Spiegels, konnten wir den Einfluss der Bewegungen des Kameragimbals zur Nachjustierung der Position zeigen. Dies hebt die Notwendigkeit einer guten Nachjustierung bzw. Regelung hervor, auf die in einem fertigen System geachtet werden muss.

Bei der vorgestellten Messmethode wurde gezeigt, dass zwischen kompensierbaren und nicht kompensierbaren Turbulenzen unterschieden werden muss. Kompensierbar sind lediglich Turbulenzen, durch die sowohl der Referenzals auch der Messstrahl geführt werden. Diesbezüglich haben wir einen zweiten möglichen Ansatz mit einem schwingungsgedämpften Referenzreflektor vorgestellt. Für die Kompensation einiger Störungen, insbesondere der Vibration durch die Rotationsfrequenz der Drohne, könnte anstelle der optischen Interferenz des Mess- und Referenzstrahls eine elektronische Verarbeitung vorteilhaft sein.

Mit dem vorgestellten System aus Abb. 1 lässt sich der Störeffekt der Drohne bereits halbieren und auftretende Turbulenzen auf dem Weg zur Drohne weitgehend unterdrücken. Es sollte dabei auf möglichst kurze Distanzen von der Drohne zum Messobjekt geachtet werden, um die dort auftretenden, nicht kompensierbaren Turbulenzen möglichst gering zu halten und Laufzeitunterschiede der Strahlen zu minimieren.

Danksagung: Die vorgestellten Arbeiten wurden von der Deutschen Forschungsgemeinschaft (DFG) im Rahmen des Projekts Chemische Expansion von Praseodym-CerMischoxidschichten bei hohen Temperaturen finanziert. Förderkennzeichen RE3980/3-1.

\section{Literatur}

[1] X. Cao und C. Rembe. Vibrationsbasierende white-etchingcracks-detektion an wälzlagern. tm - Technisches Messen, 85 (6):443-453, 2018.

[2] L. Drain. The Laser Doppler Technique. John Wiley \& Sons Ltd., Chichester,UK, 1986.

[3] B. Halkon und S. Rothberg. Towards laser doppler vibrometry from unmanned aerial vehicles. Journal of Physics: Conference Series, 1149:012022, Aug. 2018.

[4] M. Schewe, D. Kohlmann, H. Wulfmeier, H.Fritze und C. Rembe. Methoden zur minimierung des rauscheinflusses durch hitzeflimmern bei einem heterodynen laser-dopplervibrometer. tm - Technisches Messen, 87(7):443-453, 2020.

[5] M. Schewe, M. Ismail und C. Rembe. Toward airborne laser doppler vibrometry for structural health monitoring of large and curved structures. Insight - Non-Destructive Testing and Condition Monitoring Journal, The British Institute of Non-Destructive Testing, 63(5), 2021. 